\title{
The Development of a Cognitive Work Analysis Tool
}

\author{
Daniel P. Jenkins ${ }^{1}$, Neville A. Stanton ${ }^{1}$, Paul M. Salmon ${ }^{1}$, Guy H. Walker ${ }^{1}$, \\ Mark S. Young ${ }^{1}$, Ian Whitworth ${ }^{2}$, Andy Farmilo ${ }^{2}$, and Geoffrey Hone ${ }^{2}$ \\ ${ }^{1}$ School of Engineering and Design, Brunel University, Uxbridge Middlesex, UB8 3PH \\ ${ }^{2}$ Department of Information Systems, Cranfield University at the UK Defence Academy, \\ Shrivenham, SN6 8LA, UK \\ daniel.jenkins@brunel.ac.uk
}

\begin{abstract}
Due to their complexity, systems used within Network Centric Warfare and Command and Control are notoriously difficult to predict. These systems are often influenced by an ever increasing number of dynamic constraints. This dynamic instability causes problems for many traditional normative Human Factors techniques. Cognitive Work Analysis (CWA) is a formative process that focuses on these constraints rather than prescriptive methods of working; this constraint based approach allows the model to handle the unexpected and unanticipated events common in network-centric warfare. This paper presents the development of a Cognitive Work Analysis (CWA) software tool. The tool has two main purposes. The primary purpose is to assist the user in developing the large number of graphical representations that support the iterative design process. The secondary purpose is to explain CWA to novices and systematically guide them through the analysis process. The paper provides a brief introduction to CWA along with a description of the tool and its current capabilities.
\end{abstract}

Keywords: Cognitive Work Analysis; Tools; Software Development; Training.

\section{Introduction}

Cognitive Work Analysis (CWA) is a structured framework for considering the development and analysis of complex socio-technical systems. The framework leads the analyst to consider the environment the task takes place within and the effect of the imposed constraints on the system's ability to perform its purpose. The analysis guides the analyst through the process of answering the questions: why the system exists, what activities are conducted within the domain as well as how this activity is achieved, and who is performing it. Fidel \& Peijtersen (2005) describe CWA as focusing simultaneously on: the task actors perform, the environment in which it is carried out, and the perceptual, cognitive, and ergonomic attributes of the people who conduct the task.

This constraint based approach separates CWA from many other Human Factors methods currently in wide use; according to Sanderson (2003) "CWA does not focus on how human-system interaction should proceed (normative modelling) or how human-system interaction currently works (descriptive modelling). Instead, it focuses on identifying properties of the work environment and of the workers themselves that 
define possible boundaries on the ways that human-system interaction might reasonably proceed without explicitly identifying specific sequences of actions (formative modelling)." Naikar (in press) points out that by focusing on constraints, rather than on particular ways of working, CWA aims to support workers in adapting their behaviour online, in real time within system constraints, allowing them to maintain performance and safety in a variety of situations including unanticipated events. Naikar and Lintern (2002) describe the framework as supporting revolutionary rather than evolutionary design; rather than focusing on how we use new technologies to execute our current tactics and doctrine better, CWA, through its focus on constraints, aims to determine how the new technologies enable us to do things differently. Traditional methods allow evolutionary incremental improvement. It is only through formative thinking based on approaches such as CWA that exponential improvements can be realised.

The CWA framework is attracting increasing levels of interest in a wide range of socio-technical domains including, aviation (e.g., Naikar \& Sanderson, 2001); process control (e.g., Vicente, 1999); nuclear power (e.g., Olsson \& Lee, 1994); Naval (e.g., Bisantz et al, 2003); military command and control (e.g., Jenkins et al, in press); road transport (e.g., Salmon et al, In Press); health care (e.g., Miller, 2004); air traffic control (e.g., Ahlstrom, 2005); and manufacturing (e.g., Higgins, 1998). The framework has been used in system modelling (e.g. Hajdukiewicz, 1998); system design (e.g. Bisantz et al, 2003); training needs analysis (e.g. Naikar \& Sanderson, 1999), training program evaluation and design (e.g. Naikar \& Sanderson, 1999); interface design and evaluation (Vicente, 1999); information requirements specification (e.g. Ahlstrom, 2005); tender evaluation (Naikar \& Sanderson, 2001); team design (Naikar et al, 2003); and error management strategy design (Naikar \& Saunders, 2003).

By using a suite of tools (see Fig.1) the framework models the system from five different perspectives defined by Vicente (1999).

According to Naikar (2006) the first phase, Work Domain Analysis, identifies the constraints on workers' behaviour that are imposed by the purposive and physical context, or problem space, in which workers operate. Work Domain Analysis is used to define the task environment. It identifies a fundamental set of constraints on the actions of any actor, thus providing a solid foundation for subsequent phases. The system domain is represented at a number of conceptual levels. At the highest level the system's raison d'être is represented; at the lowest level the physical objects within the system are described.

The second phase, Control Task Analysis, is used to understand activity within the domain; it allows us to identify the requirements associated with known, recurring classes of situations. This phase specifies the input and end goal leaving a 'black box' in the middle. The phase identifies what needs to be done independently of how or by whom.

Strategies Analysis, the third phase of CWA looks at filling in the 'black box' left in control task analysis; it looks at different ways of carrying out the same task. Wherever the previous phase dealt with the question of what needs to be done this phase addresses how it can be done.

The fourth phase, Social Organisation \& Cooperation Analysis, addresses dividing the task between the available resources and looks at how the team communicates and 
cooperates. The objective is to determine how the social and technical factors in a socio-technical system can work together in a way that enhances the performance of the system as a whole.

The fifth and final phase, Worker Competencies Analysis, focuses on the types of behaviour required by workers to conduct a task. This section addresses the more traditional core concerns of the Human Factors and HCI communities.

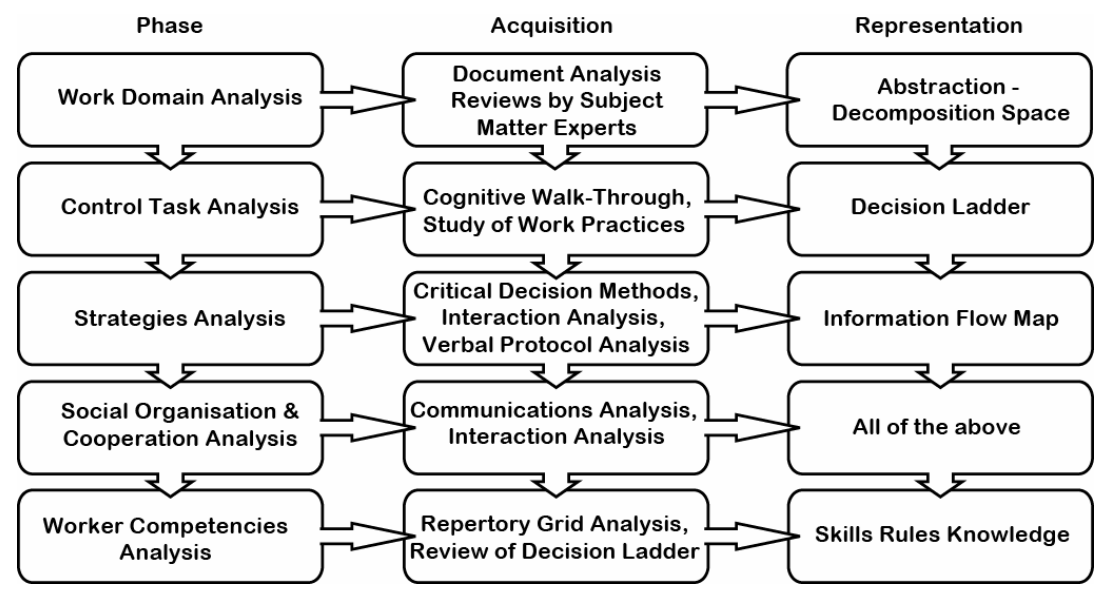

Fig. 1. The five phases of CWA according to Vicente (1999) (Acquisition methods added from Lintern et al (2004))

\section{The Tool}

The CWA process is often criticised for being complex and time consuming (Cummings, 2006); the tool presented in this paper attempts to provide some level of guidance and expedite the documentation process in each of the five phases identified by Vicente (1999) to address these concerns. In order to provide guidance the tool uses an example scenario of an experimental sensor to effecter paradigm (Jenkins et al, in press) to describe and illustrate the process.

The approach taken by an analyst conducting a CWA is highly dependent on the domain and the aims of the research. Many CWA analyses will not focus on all of the five phases; the majority of analyses place a much greater focus on the first phase, Work Domain Analysis. The type of analysis conducted is likely to depict which of the phases are used and in what order and ratio. Interface design such as 'Ecological Interface Design' (EID) (Burns \& Hajdukiewicz, 2004) tends to use only the first phase (Work Domain Analysis) when designing new interfaces. An analysis of team design or training needs is more likely to focus more heavily on Control Task Analysis and Social Organisational and Cooperation Analysis as these phases capture activity and consider its distribution amongst the system's assets. The benefit of the framework is that the CWA approach can be applied throughout the system life cycle for the design, development, representation and evaluation of both conceptual and existing operational systems. In order to support this often non-linear process the tool 
has been designed with a tabbed bar built into the interface, this tabbed bar allows novices to approach the framework in a linear process whilst allowing users to jump backwards and forwards at their own convenience. Each of the phases is decomposed into a number of sections (see Fig. 2). The first section introduces the phase in generic terms briefly explaining the principles behind the particular level of the framework. The next section provides a worked example of the analysis at the current phase consistently using the sensor to effecter case study (Jenkins et al, in press) The example is fully annotated in an attempt to provide guidance to novices, and to provide an idea of the finished product. The subsequent sections allow the user to construct and develop the models and documentation required; the process is supported by mouse-overs and prompts to guide novices in creating diagrams.

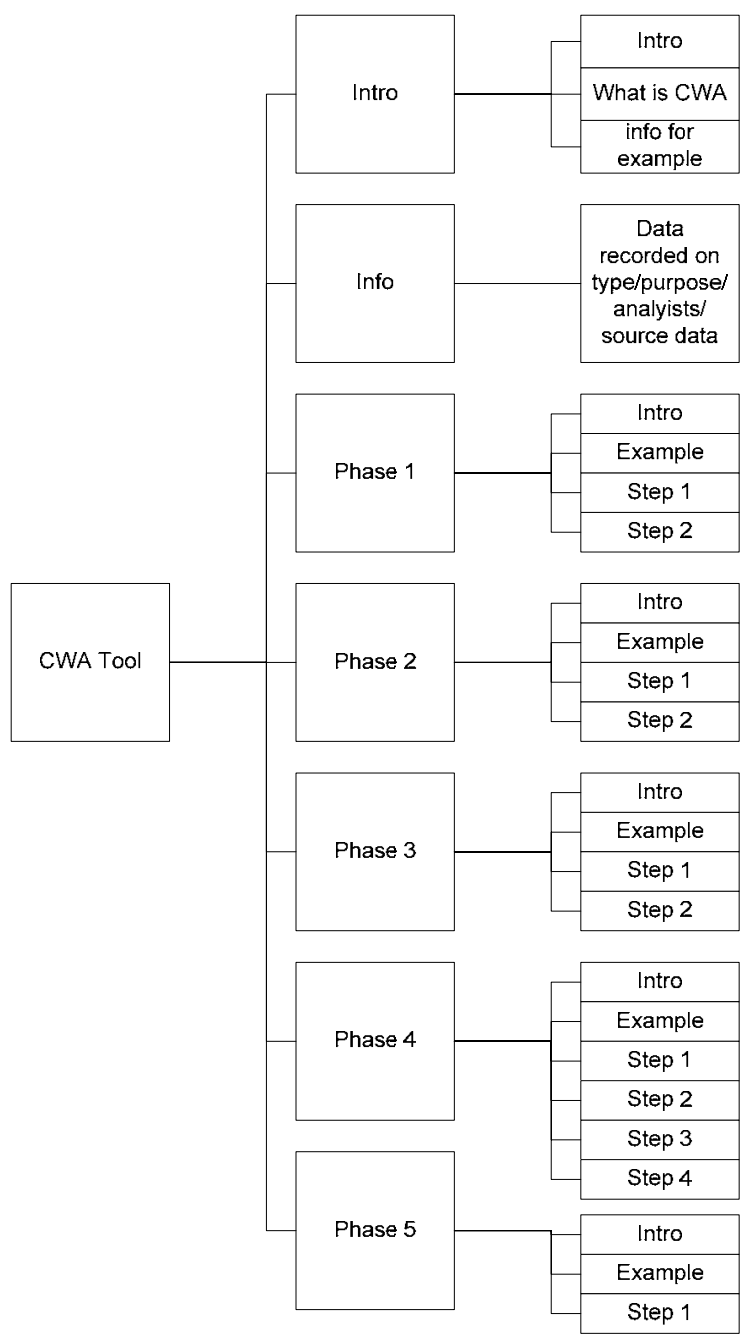

Fig. 2. Map of tool function 


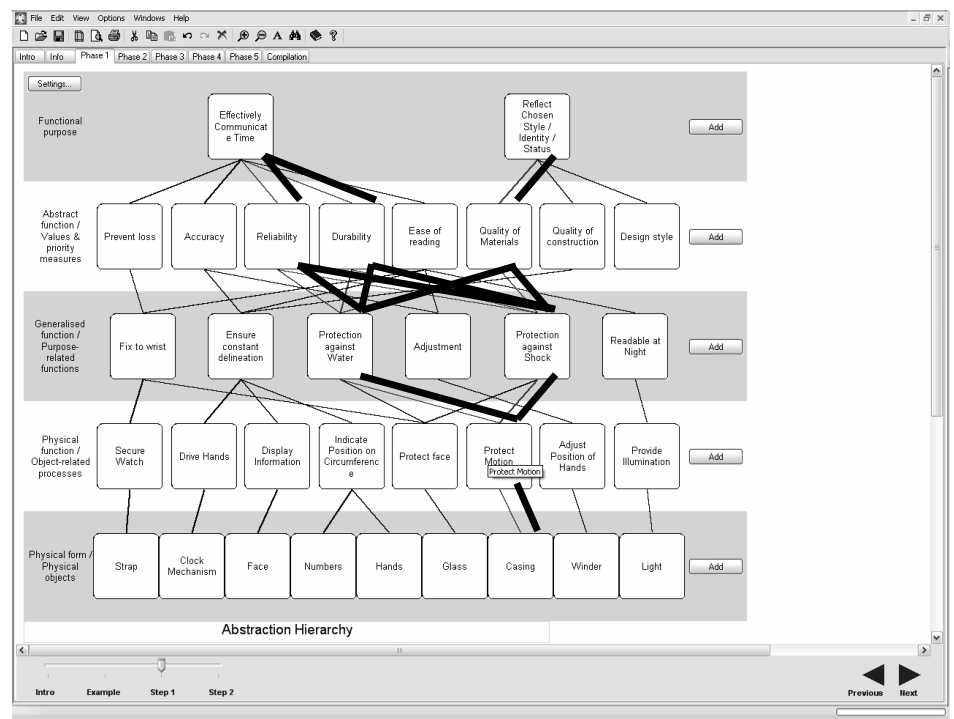

Fig. 3. Example of Abstraction Hierarchy created by the tool

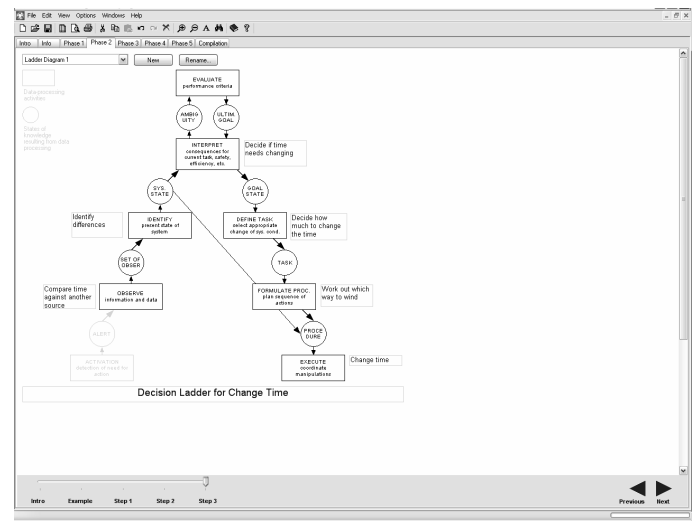

Fig. 4. Example of Contextual Activity Template created by the tool

The dynamic nature of the software tool has many benefits over its paper based counterpart; allowing files to be saved, copied, edited and rapidly transmitted. Feature such as an ability to export all of the products to a Word document with a single button click also make the report compiling process much simpler. Diagrams are made far more manageable by the tool facilitating the ability to rapidly reorder the diagrams and representations. The tool has been intentionally designed to be unconstrained allowing researches to apply their own interpretations of the framework, although in order to constrain novices from making fundamental mistakes some of the functions are deliberately not permitted, such as connecting to data processing activities in the decision ladder or connecting a node in the abstraction hierarchy to a node two levels above. 


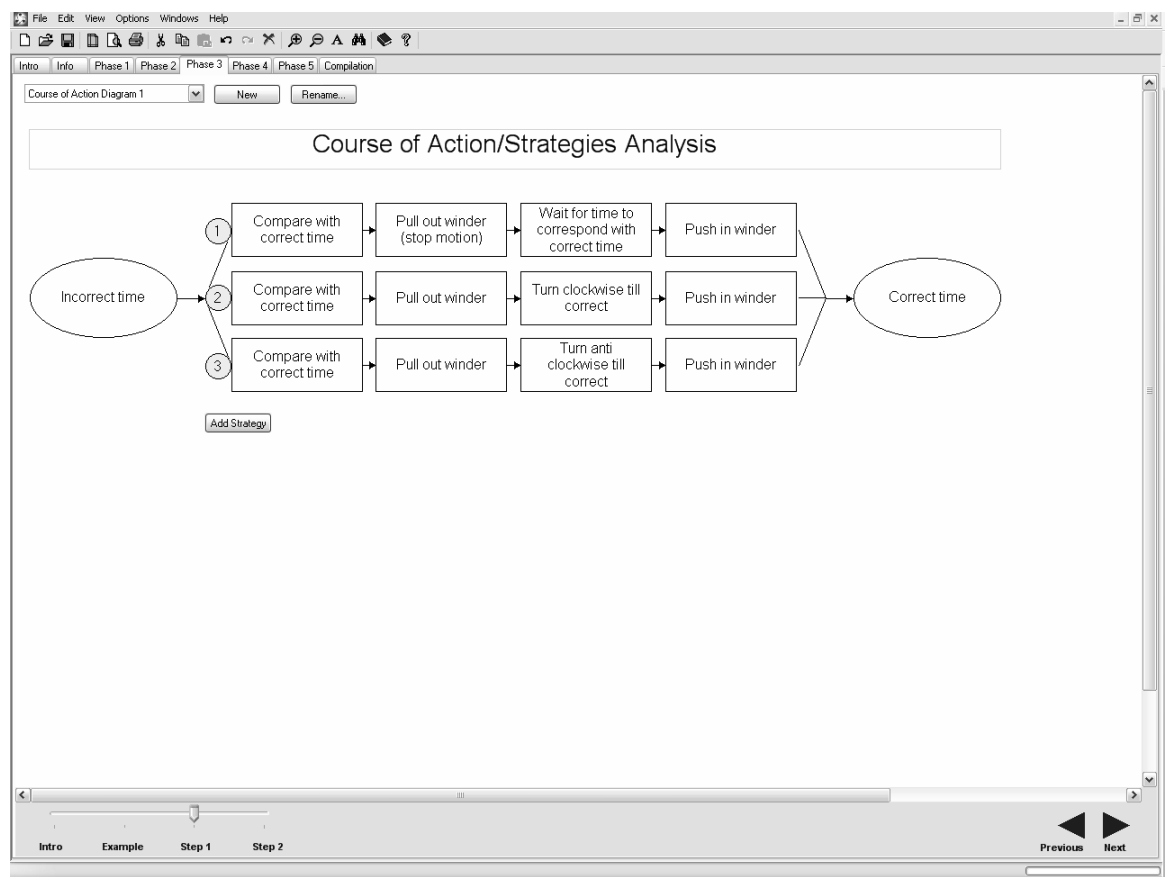

Fig. 5. Example of Strategies Analysis created by the tool

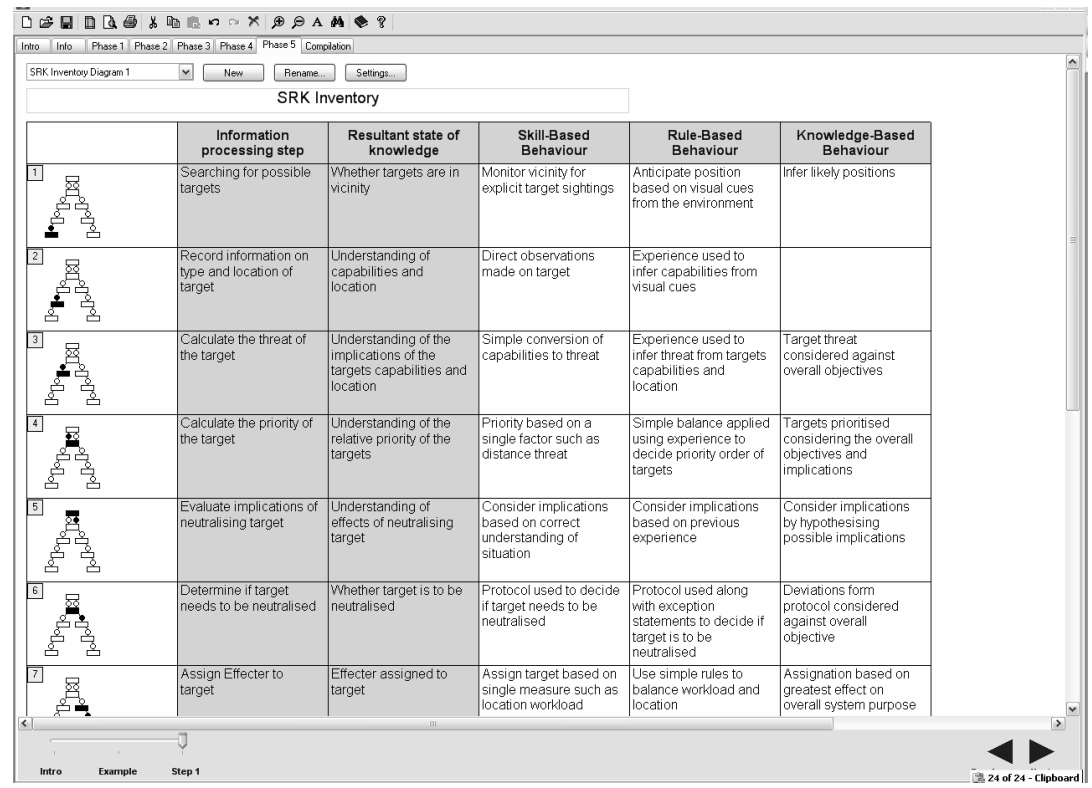

Fig. 6. Example of Skills Rules and Knowledge Table created by the tool 
Additional benefits from the dynamic nature of the tool have also been exploited: When the user places their mouse over a node on the Abstraction Hierarchy the linked nodes both up and down the hierarchy are highlighted in red (for illustrative purpose these have been shown in bold in); this allows a cause and effect relationship to be examined within the different levels. The speed in which the abstraction hierarchy can be developed, edited and reviewed (helped by the addition of the red links) makes it feasible to carry out the generation and validation of representation from scratch with Subject Matter Experts (SMEs) in real time.

The software tool passes important data forward aiding the completion of subsequent representations. This significantly reduces the tedium of the documentation process. This also means that minor changes to text semantics and diagram layout is fed through from the initial stages to the subsequent phases. This has particular benefits in the Social Organisation and Cooperation phase, which reuses the products generated in the previous three phases. This semi structured process combined with the ease of documentation further encourages analysts to continue their analysis beyond the first two phases of CWA, a concern raised by Cummings (2006).

\section{Conclusions}

This paper has briefly introduced CWA along with a short description of the software tool and its potential benefits.

Whilst the tool is still in its development stages some of the benefits in terms of novice training and its ability to expedite the documentation process are already clearly visible. The dynamic nature of the tool has also revealed some unanticipated benefits in eliciting domain information from subject matter experts.

The development of the tool now continues with a global structured feedback process led by some of the leading researchers in CWA.

Acknowledgement. This research from the Human Factors Integration Defence Technology Centre was part-funded by the Human Sciences Domain of the UK Ministry of Defence Scientific Research Program.

Any views expressed are those of the authors and do not necessarily represent those of MOD or any other UK government department.

\section{References}

1. Ahlstrom, U.: Work domain analysis for air traffic controller weather displays. Journal of Safety Research 36, 159-169 (2005)

2. Bisantz, A.M., Roth, E., Brickman, B., Gosbee, L.L., Hettinger, L., McKinney, J.: Integrating cognitive analyses in a large-scale system design process. International Journal of Human-Computer Studies 58, 177-206 (2003)

3. Burns, C.M., Hajdukiewicz, J.R.: Ecological Interface Design. CRC Press, Boca Raton, FL (2004)

4. Cummings, M.L.: Can CWA inform the design of networked intelligent systems. Moving Autonomy Forward Conference 2006, Lincoln, UK (2006) 
5. Fidel, R., Pejtersen, A.M.: Cognitive Work Analysis. In: Fisher, K.E., Erdelez, S., McKechnie, E.F. (eds.) Theories of information behavior: A researcher's guide, Information Today, Medford, NJ (2005)

6. Hajdukiewicz, J.R.: Development of a structured approach for patient monitoring in the operating room. Masters Thesis. University of Toronto (1998)

7. Higgins, P.G.: Extending cognitive work analysis to manufacturing scheduling. In: Proceedings of OzCHI'98, November 30-December vol. 4, pp. 236-243 (1998)

8. Jenkins, D.P., Stanton, N.A., Salmon, P.M, Walker, G.H., Young, M.S.: Cognitive Work Analysis of a Sensor to Effecter System: Implications for Network Structures. Human Factors and Ergonomics Society Europe Chapter Meeting 2006 (in Press)

9. Lintern, G., Cone, S., Schenaker, M., Ehlert, J., Hughes, T.: Asymmetric Adversary Analysis for Intelligent Preparation of the Battlespace (A3-IPB) United States Air Force Research Department Report (2004)

10. Miller, A.: A work domain analysis framework for modelling intensive care unit patients. Cognition, Technology and Work. 6(4), 207-222 (2004)

11. Naikar, N.: Modelling activity in network-centric operations with cognitive work analysis: Work situations, work functions, decisions, and strategies. In: Bolia, B. (ed.) Supporting decision effectiveness in network-centric operations. Wright Patterson Air Force Base, Dayton, $\mathrm{OH}$ (in press)

12. Naikar, N.: Beyond interface design: Further applications of cognitive work analysis. International Journal of Industrial Ergonomics 36, 423-438 (2006)

13. Naikar, N., Lintern, G.: A review of Cognitive Work Analysis: Towards safe, productive, and healthy computer-based work by Vicente, K.J. The International Journal of Aviation Psychology, 12(4), 391-400 (2002)

14. Naikar, N., Sanderson, P.M.: Work domain analysis for training-system definition. International Journal of Aviation Psychology 9, 271-290 (1999)

15. Naikar, N., Sanderson, P.M.: Evaluating design proposals for complex systems with work domain analysis. Human Factors 43, 529-542 (2001)

16. Naikar, N., Saunders, A.: Crossing the boundaries of safe operation: A technical training approach to error management. Cognition Technology and Work. 5, 171-180 (2003)

17. Olsson, G., Lee, P.L.: Effective interfaces for process operators. The. Journal of Process Control 4, 99-107 (1994)

18. Salmon, P.M., Stanton, N.A., Regan, M., Lenne, M., Young, K.: Work domain analysis and road transport: Implications for vehicle design. International Journal of Vehicle Design (In Press)

19. Sanderson, P.M.: Cognitive Work Analysis across the system life-cycle: Achievements, challenges, and prospects in aviation. In: Pfister, P., Edkins, G. (eds.) Aviation Resource Management (Vol 3). Aldershot, UK Ashgate (2003)

20. Vicente, K.J.: Cognitive work analysis: Toward safe, productive, and healthy computerbased work. Lawrence Erlbaum Associates, Mahwah, NJ (1999) 